\title{
Participação das famílias em Programas de Saúde Auditiva: um estudo descritivo
}

\author{
Families participation in Hearing Health Programs: \\ a descriptive study
}

\author{
Kátia de Freitas Alvarenga ${ }^{1}$, Maria Cecília Bevilacqua ${ }^{2}$, Tatiana Mendes de Melo ${ }^{3}$, \\ Andréa Cintra Lopes ${ }^{4}$, Adriane Lima Mortari Moret ${ }^{5}$
}

\begin{abstract}
RESUMO
Objetivo: Avaliar a adesão dos pais para a realização dos procedimentos auditivos em um Programa de Saúde Auditiva Infantil, desenvolvido na comunidade. Métodos: Foi realizada uma análise comparativa das informações sobre o comparecimento nos atendimentos de dois Programas de Saúde Auditiva Infantil, um vinculado a um hospital público e outro realizado junto à Estratégia Saúde da Família (ESF). A casuística foi formada por 362 crianças, nascidas entre o período de fevereiro a março de 2007 no Hospital em que é realizada a triagem auditiva, e que foram agendadas para participar do Programa junto a ESF. Resultados: Das 362 crianças que nasceram neste hospital e que foram encaminhadas para realização da avaliação audiológica na unidade básica de saúde, 147 compareceram ao atendimento, representando $40,61 \%$ de adesão das famílias no projeto desenvolvido na comunidade. Dos casos com resultado de triagem auditiva sugestivo de ausência de alteração auditiva, somente 39,80\% aderiram ao Programa realizado junto ao ESF. Dos casos que não completaram alguma etapa do processo de identificação na maternidade, 32 (55,18\%) famílias não aderiram a nenhum dos Programas de Saúde Auditiva existentes, ficando sem esclarecimento sobre a audição do filho. Conclusão: O comparecimento das famílias nas UBS foi inferior à metade das famílias que foram convidadas a levar os filhos para a avaliação audiológica, independentemente de se a criança havia sido submetida ao processo de identificação da deficiência auditiva na maternidade pública da cidade.
\end{abstract}

Descritores: Audição; Perda auditiva; Triagem neonatal; Saúde da família; Sistema Único de Saúde; Planos e Programas de Saúde

\section{INTRODUÇÃO}

Nos últimos anos, no Brasil, a deficiência auditiva na infância vem sendo discutida no âmbito da saúde pública a fim de determinar e programar ações em saúde auditiva que permitam prevenir, identificar, diagnosticar e proporcionar a reabilitação auditiva em crianças com deficiência auditiva

Trabalho realizado no Curso de Fonoaudiologia da Faculdade de Odontologia de Bauru, Universidade de São Paulo - USP - Bauru (SP), Brasil.

(1) Curso de Fonoaudiologia da Faculdade de Odontologia de Bauru, Universidade de São Paulo - USP - Bauru (SP), Brasil.

(2) Curso de Fonoaudiologia da Faculdade de Odontologia de Bauru, Universidade de São Paulo - USP - Bauru (SP), Brasil.

(3) Curso de Fonoaudiologia, Universidade de Guarulhos - UNG - Guarulhos (SP), Brasil.

(4) Curso de Fonoaudiologia da Faculdade de Odontologia de Bauru, Universidade de São Paulo - USP - Bauru (SP), Brasil.

(5) Curso de Fonoaudiologia da Faculdade de Odontologia de Bauru, Universidade de São Paulo - USP - Bauru (SP), Brasil.

Endereço para correspondência: Kátia de Freitas Alvarenga. Faculdade de Odontologia de Bauru - Universidade de São Paulo. Al. Dr. Octávio Pinheiro Brisolla, 9-75, Vila Universitária, Bauru (SP), Brasil, CEP: 17012-101. E-mail: katialv@fob.usp.br

Recebido em: 15/6/2009; Aceito em: 12/08/2009 de origem congênita ou adquirida.

Com o objetivo de reduzir a idade no diagnóstico e no início da intervenção na deficiência auditiva na infância, vários programas têm sido propostos, entre eles, a Triagem Auditiva Neonatal (TAN). A meta da identificação e intervenção precoces é maximizar o desenvolvimento da função auditiva, possibilitando a competência linguística e, também, o desenvolvimento acadêmico das crianças portadoras de deficiência auditiva, uma vez que a criança deficiente auditiva que não recebe oportunidades apropriadas para adquirir a linguagem oral pode apresentar atraso no desenvolvimento linguístico, cognitivo e sócio-emocional ${ }^{(1)}$. Os benefícios desta ação precoce estão presentes independentemente do gênero, nível sócio-econômico e forma de comunicação utilizada $^{(2)}$.

Para atingir tais benefícios a triagem auditiva neonatal deve estar inserida em Programas de Saúde Auditiva Neonatal, o que permitirá que todas as etapas recomendadas para a identificação e intervenção nos primeiros meses de vida sejam cumpridas $^{(1)}$.

Ao considerar-se que a avaliação audiológica, nos primeiros meses de vida, requer profissionais especializados e equi- 
pamentos sofisticados, condições oferecidas pelos Centros de Referência de Alta Complexidade referentes ao atendimento da pessoa com deficiência auditiva ${ }^{(3)}$, faz-se necessário criar um fluxo contínuo de acolhimento para que as famílias tenham acesso rápido e fácil a eles. Não criar estas condições para a família, significa na maioria das vezes, deixá-las sem esclarecimento sobre a audição do seu filho, uma vez que a população beneficiada pelo Sistema Único de Saúde (SUS) geralmente não apresenta recursos financeiros e sociais para procurar atendimento no sistema privado.

Contudo, mesmo com programas efetivos de TAN, ainda existe uma porcentagem de recém nascidos que não realizam a triagem auditiva, tanto a etapa teste quanto a de re-teste; há também uma taxa elevada de evasão dos neonatos, que não dão continuidade às outras etapas do processo de identificação e intervenção, sendo tal situação apontada como uma das dificuldades dos programas de TAN tanto em nível nacional quanto internacional ${ }^{(4-12)}$. Este fato demonstra a necessidade de ser formada uma rede de identificação, diagnóstico, intervenção, orientação e apoio às famílias.

Inúmeras situações são apontadas como razões para o alto índice de evasão nos Programas de TAN (teste ou re-teste da triagem auditiva e diagnóstico), tais como: falta de disponibilidade dos pais em trazer seus filhos para a realização da avaliação audiológica; pouco conhecimento por parte da família sobre a importância da detecção e intervenção precoce na deficiência auditiva, bem como as concepções, baseadas no senso comum, que os pais fazem sobre a audição de seus filhos, como o pensamento de que se a criança pode detectar os sons ambientais, eles podem ouvir e desenvolver normalmente a fala e linguagem ${ }^{(7,10-13)}$.

A orientação à família é imprescindível, portanto, para que não haja desistência no decorrer do processo, devendo assim, ser contínua e abordar todos os aspectos que permitam a família compreender e valorizar o atendimento que está sendo realizado ${ }^{(14)}$.

Analisando a problemática exposta, fica evidente a necessidade da criação de ações que ampliem a identificação e a detecção da deficiência auditiva nos primeiros meses de vida em todos os níveis de atenção à saúde, e não apenas na TAN.

No Brasil, a Saúde da Família representa a estratégia de reorientação do modelo de atenção básica à saúde, antes centrado na assistência médica curativa, para um modelo centrado na promoção e prevenção da saúde. Em Bauru, interior do estado de São Paulo, o início da mudança do modelo assistencial ocorreu em fevereiro de 2000, a partir da implantação do Programa de Agentes Comunitários de Saúde (PACS) no núcleo de saúde do bairro Jardim Godoy. Em 2002, a primeira equipe Saúde da Família iniciou as atividades no bairro Santa Edwirges e posteriormente na Vila São Paulo. Atualmente, o município apresenta um PACS e sete equipes de Saúde da Família conferindo cobertura a $6,79 \%$ da população da cidade ${ }^{(15)}$.

No âmbito da saúde auditiva, no ano de $2003^{(16)}$, também na cidade de Bauru, foi implantado o Programa de Saúde Auditiva Neonatal na maternidade pública da cidade - Maternidade Santa Isabel.
A orientação familiar é um dos aspectos mais discutidos neste Programa e é realizada em todas as etapas do atendimento, inclusive antes da realização da triagem auditiva, com a mãe ainda no leito hospitalar. No decorrer desses anos, a triagem auditiva neonatal no Hospital Maternidade tem mantido taxa superior a $90 \%$ dos recém-nascidos/mês, porém não alcançando os $100 \%$. Além disso, ainda se registra a ocorrência de desistências da família durante o processo de identificação ou diagnóstico ${ }^{(16)}$.

Neste contexto, visando à continuidade do Programa de Saúde Auditiva Neonatal, permitindo assim, resgatar essas crianças e acompanhar o seu desenvolvimento em audição e linguagem no primeiro ano de vida, foi implantado o Projeto Modelo de Saúde Auditiva Infantil no Programa de Saúde da Família (SAÚDI) ${ }^{(17)}$, que propõe a atuação dos Agentes Comunitários de Saúde (ACS) na área de saúde auditiva. Os objetivos do projeto foram a capacitação dos ACS na área de saúde auditiva, e a validação de um instrumento a ser utilizado por esses profissionais nas visitas domiciliares, para o acompanhamento do desenvolvimento da audição e da linguagem da criança. Ambos os Programas foram desenvolvidos em parceria com a Secretaria Municipal de Bauru, São Paulo.

O objetivo do presente estudo foi avaliar a adesão dos pais a um Programa de Saúde Auditiva Infantil vinculada à comunidade, no município de Bauru, estado de São Paulo.

\section{MÉTODOS}

Trata-se de um estudo observacional, do tipo transversal, no qual a população estudada foi composta por crianças de zero a um ano de idade, nascidas em uma Maternidade Pública do município e acompanhadas pelo PACS ou por equipes de Saúde da Família que participaram do Programa de Capacitação em Saúde Auditiva proposto no projeto SAÚDI ${ }^{(17)} \mathrm{em}$ três diferentes bairros da cidade de Bauru (SP).

\section{Casuística}

Como parte deste projeto, foram agendadas para avaliação audiológica nas unidades básicas de saúde (UBS) de referência do PACS e das equipes de Saúde da Família, entre o período de fevereiro a março de 2007, 418 crianças de zero a um ano de idade, correspondendo ao total de crianças atendidas nesta faixa etária pelos Agentes Comunitários de Saúde (ACS) das equipes descritas. A avaliação audiológica foi realizada, a fim de validar o questionário de acompanhamento do desenvolvimento da audição e da linguagem da criança em crianças de zero a um ano, para sua posterior utilização pelas equipes de Saúde da Família.

Contudo, a casuística deste estudo foi formada por 362 crianças que nasceram na Maternidade citada, e foram agendadas para participar do Projeto SAÚDI. Crianças acima da faixa etária estabelecida e que não nasceram na referida Maternidade foram excluídas da casuística. É digno de nota que a escolha dessa Maternidade para participação no presente estudo foi determinada pelo fato de ser a única maternidade pública do município que oferece a TAN. 


\section{Procedimento}

O agendamento do atendimento na UBS foi realizado pelos ACS durante sua primeira visita domiciliar voltada à saúde auditiva, na qual o questionário de acompanhamento do desenvolvimento da audição e da linguagem da criança foi aplicado pelos profissionais. Neste questionário, além de questões relacionadas aos dados pessoais da criança e informações sobre o desenvolvimento das funções auditiva e de linguagem, havia a seguinte pergunta: "Em que Maternidade a criança nasceu?", para que, posteriormente, pudessem ser confrontados os dados sobre o comparecimento da mesma criança, no Programa de Saúde Auditiva Neonatal da maternidade pública e no Projeto SAÚDI ${ }^{(17)}$.

O Programa de Saúde Auditiva Neonatal da maternidade pública possui um banco de dados, no qual são inseridas as informações sobre as diversas etapas: triagem auditiva neonatal realizada em um Hospital Maternidade; diagnóstico e reabilitação em um serviço de Audiologia habilitado como Alta Complexidade pelo Ministério da Saúde. Assim, foi possível verificar que das 418 crianças agendadas para avaliação audiológica no Projeto SAÚDI, 362 haviam nascido na referida Maternidade. Desta forma, os dados referentes à adesão das famílias aos atendimentos audiológicos na Maternidade e/ou serviço de Audiologia (Programa Modelo de Saúde Auditiva no Recém-nascido) e nas UBS (Projeto SAÚDI) foram confrontados. Neste estudo a adesão foi caracterizada pelo índice de comparecimento, em porcentagem, no atendimento no núcleo de saúde.

Cabe ressaltar que a avaliação audiológica proposta pelo projeto SAÚDI ${ }^{(17)}$ foi realizada por Fonoaudiólogo, além da avaliação otorrinolaringológica, nos Núcleos de Saúde de referência para a família. O dia da semana definido foi o sábado, das oito às 17 horas, devido à maior disponibilidade de salas nos núcleos, e à experiência prévia da Secretaria da Saúde com programas desenvolvidos anteriormente, nos quais houve maior adesão da comunidade quando os mesmos foram realizados aos finais de semana.

\section{Análise estatística}

Os dados do estudo foram armazenados em banco de dados do Excel e foi realizada a análise descritiva, por meio dos resultados, em porcentagem.

\section{Aspectos éticos}

O presente estudo foi aprovado pelo Comitê de Ética em Pesquisa da Faculdade de Odontologia de Bauru da Universidade de São Paulo, sob protocolo número 73/2006. Foram seguidos, na condução do estudo, os aspectos éticos pertinentes.

\section{RESULTADOS}

Das 362 crianças que nasceram no Hospital Maternidade, e que foram encaminhadas para realização da avaliação audiológica na UBS, 147 compareceram ao atendimento, representando 40,61\% de adesão das famílias ao projeto desenvolvido na comunidade (Tabela 1).
Tabela 1. Comparecimento da casuística estudada no Programa SAÚDI

\begin{tabular}{lcccccc}
\hline & \multicolumn{2}{c}{ Sim } & \multicolumn{2}{c}{ Não } & \multicolumn{2}{c}{ Total } \\
& N & $\%$ & N & $\%$ & N & $\%$ \\
\hline Santa Edwirges & 73 & 34,76 & 137 & 65,24 & 210 & 100 \\
Jardim Godoy & 27 & 54 & 23 & 46 & 50 & 100 \\
Vila São Paulo & 47 & 46,08 & 55 & 53,92 & 102 & 100 \\
Total & 147 & 40,61 & 215 & 59,39 & 362 & 100 \\
\hline
\end{tabular}

Posteriormente foram verificadas as informações sobre o comparecimento das mesmas famílias na triagem auditiva neonatal realizada no Programa Modelo de Saúde Auditiva no recém-nascido ${ }^{(16)}$, de acordo com o núcleo de saúde (Tabela 2).

Tabela 2. Comparecimento da casuística estudada no programa de triagem auditiva neonatal oferecido na Maternidade

\begin{tabular}{lcccccc}
\hline & \multicolumn{2}{c}{ Sim } & \multicolumn{2}{c}{ Não } & \multicolumn{2}{c}{ Total } \\
& N & $\%$ & N & $\%$ & N & $\%$ \\
\hline Santa Edwirges & 175 & 83,33 & 35 & 16,67 & 210 & 100 \\
Jardim Godoy & 43 & 86 & 7 & 14 & 50 & 100 \\
Vila São Paulo & 86 & 84,31 & 16 & 15,69 & 102 & 100 \\
Total & 304 & 83,98 & 58 & 16,02 & 362 & 100 \\
\hline
\end{tabular}

A Tabela 3 apresenta os resultados referentes ao comparecimento das famílias que realizaram a triagem auditiva na maternidade (com resultado sugestivo de audição normal) no atendimento marcado na UBS.

Tabela 3. Comparecimento das crianças com resultado passa na triagem auditiva neonatal no Programa SAÚDI

\begin{tabular}{lcccccc}
\hline & \multicolumn{2}{c}{ Sim } & \multicolumn{2}{c}{ Não } & \multicolumn{2}{c}{ Total } \\
& N & $\%$ & N & $\%$ & N & $\%$ \\
\hline Santa Edwirges & 59 & 33,71 & 116 & 66,29 & 175 & 100 \\
Jardim Godoy & 23 & 53,49 & 20 & 46,51 & 43 & 100 \\
Vila São Paulo & 39 & 45,35 & 47 & 54,65 & 86 & 100 \\
Total & 121 & 39,80 & 183 & 60,20 & 304 & 100 \\
\hline
\end{tabular}

Os resultados referentes à adesão das famílias que não realizaram alguma etapa do processo de identificação no Programa oferecido pela maternidade (teste, re-teste ou diagnóstico audiológico) no atendimento marcado na UBS podem ser visualizados na Tabela 4.

Tabela 4. Comparecimento das crianças que não realizaram a triagem auditiva neonatal no Programa SAÚDI

\begin{tabular}{lcccccc}
\hline & \multicolumn{2}{c}{ Sim } & \multicolumn{2}{c}{ Não } & \multicolumn{2}{c}{ Total } \\
& N & $\%$ & N & $\%$ & N & $\%$ \\
\hline Santa Edwirges & 14 & 40 & 21 & 60 & 35 & 100 \\
Jardim Godoy & 4 & 57,15 & 3 & 42,85 & 7 & 100 \\
Vila São Paulo & 8 & 50 & 8 & 50 & 16 & 100 \\
Total & 26 & 44,82 & 32 & 55,18 & 58 & 100 \\
\hline
\end{tabular}


Do total de 58 crianças que não participaram efetivamente das etapas do Programa oferecido na Maternidade, 26 famílias (44,82\%) compareceram nos Núcleos de Saúde para avaliação audiológica após orientação do ACS. Assim, 32 famílias $(55,18 \%)$ não participaram de nenhum dos programas de saúde auditiva existentes, seja na Maternidade, seja no Núcleo de Saúde.

\section{DISCUSSÃO}

O comparecimento das famílias nas UBS foi inferior à metade das que foram convidadas a levar os filhos para a avaliação audiológica, abrangendo 40,61\% da casuística esperada (Tabela 1). Independentemente da UBS, a expectativa inicial dos profissionais envolvidos no Programa SAÚDI ${ }^{(17)}$ era de obter o comparecimento familiar de forma mais efetiva, graças às condições em que o mesmo foi desenvolvido: ausência de custo para a família; agendamento realizado pelos ACS durante a visita domiciliar; avaliação audiológica no Núcleo de Saúde do bairro em que a família reside e durante os finais de semana, o que a princípio, aumentaria a possibilidade de comparecimento, pois não haveria prejuízo no trabalho do responsável.

Este baixo índice de comparecimento das famílias para realização da avaliação audiológica nas UBS, levou a questionamentos sobre os motivos que justificariam este fato.

Correlacionando as informações do banco de dados da maternidade pública e do Projeto SAÚDI, das crianças que realizaram a triagem auditiva no Programa da Maternidade, apenas 39,80\% compareceram ao atendimento para avaliação audiológica no Núcleo de Saúde (Tabela 3). O fato de a criança já ter sido submetida à triagem auditiva neonatal, com resultado sugestivo de audição normal, pode estar associado a este índice de comparecimento no atendimento na UBS. Este achado desperta preocupação, visto que todo procedimento de triagem auditiva não apresenta $100 \%$ de sensibilidade, havendo sempre taxas de falso-negativos, assim como existe a possibilidade das perdas auditivas adquiridas e de início tardio. Mesmo com a orientação familiar após a realização da triagem auditiva sobre estas possibilidades e sobre a necessidade da família acompanhar o desenvolvimento da audição e da linguagem da criança, por vezes o resultado indicativo de audição normal no momento do nascimento pode levar a família a não se preocupar com a audição da criança, não questionando o resultado da TAN ou a mudança do quadro audiológico da criança.

Outro aspecto muito importante constatado neste estudo foi que das 58 crianças que não realizaram ou completaram a triagem auditiva neonatal realizada na Maternidade, 32 famílias não participaram de nenhum dos programas de saúde auditiva existentes, seja na Maternidade, seja no Núcleo de Saúde, ficando assim, sem esclarecimento sobre a audição do filho (Tabela 4). Semelhante resultado foi descrito em estudo anterior que constatou que existem famílias que se recusam a participar de programas voltados à audição, fato atribuído pelos autores à falta de conhecimento da população sobre aspectos voltados a essa área do desenvolvimento ${ }^{(9)}$.

Estudos sobre a implementação de programas de TAN apontaram a taxa de evasão dos neonatos, que não realizam a triagem auditiva ou a falta de continuidade das outras etapas do processo de identificação, como uma das maiores dificuldades encontradas nestes programas ${ }^{(3-11,18)}$, em consonância com as dificuldades encontradas no presente estudo.

As principais razões apontadas na literatura para justificar este fato seriam: a dificuldade de transporte à maternidade ${ }^{(12)}$, falta de horário disponível para levar o filho para realizar a triagem auditiva ${ }^{(10)}$, aceitação dos pais em participar do processo de identificação e diagnóstico da alteração auditiva ${ }^{(9)}$, grau de instrução ${ }^{(11)} \mathrm{e}$ falta de conscientização da família sobre o impacto da deficiência auditiva no desenvolvimento da criança ${ }^{(6)}$. Outros aspectos que também poderiam estar associados com a falta de comparecimento das famílias nos Programas de Saúde Auditiva Neonatal são: situação sócio-econômica, número de filhos, escolaridade e idade materna, dentre outros.

Considerando que o Programa SAÚDI ${ }^{(17)}$ foi desenvolvido no Núcleo de Saúde do bairro no qual a família reside e durante os finais de semana, parece que a falta de condições financeiras não seria uma justificativa para o não comparecimento destas famílias. A falta de conhecimento por parte da comunidade sobre a importância da audição no desenvolvimento global da criança, bem como os fatores de risco para adquirir a deficiência auditiva, pode ser o fator preponderante desta situação.

No Programa da Maternidade, após a triagem auditiva, todas as mães são orientadas pelas fonoaudiólogas sobre a importância do diagnóstico audiológico e o acompanhamento da função auditiva e do desenvolvimento de linguagem devido à possibilidade da ocorrência das perdas de audição adquiridas ou de início tardio ${ }^{(16)}$.

Desta maneira, os resultados do presente estudo parecem apontar a necessidade de maior ênfase no momento da orientação após o exame, a respeito da ocorrência das perdas de audição adquiridas ou de início tardio na população infantil, como também um trabalho de conscientização da família sobre a audição, os aspectos etiológicos da deficiência auditiva, bem como a importância do diagnóstico e do processo terapêutico precoce nesta deficiência.

Cabe ressaltar que em Bauru, a cobertura assistencial por parte dos ACS é baixa, totalizando 6,77\% da população da cidade. Contudo, em território nacional, as equipes de Saúde da Família acompanham mais de 87 milhões de brasileiros ${ }^{(19)}$. Desta forma, a atuação das equipes de Saúde da Família do Brasil, quando capacitadas adequadamente sobre saúde auditiva infantil, pode auxiliar não apenas na promoção da saúde auditiva e na detecção das desordens auditivas, como também na conscientização da comunidade sobre a importância de todas as etapas do processo de identificação e tratamento da deficiência auditiva e no resgate dos pacientes faltosos aos serviços de referência, fortalecendo assim a Política Nacional de Saúde Auditiva implantada em 2004, a partir da Portaria GM/MS n ${ }^{\circ} 2.073$ de 28/09/2004 permitirá alcançar os objetivos propostos, como diagnóstico e intervenção precoces na deficiência auditiva.

\section{CONCLUSÃO}

A taxa de comparecimento geral das famílias no programa desenvolvido na comunidade foi inferior à metade das famílias que foram convidadas a levar os filhos para a avaliação audiológica, independentemente de se a criança havia sido submetida ao processo de identificação da deficiência auditiva na maternidade pública da cidade.

A atuação dos ACS das equipes da Saúde da Família vem 
assumir papel fundamental não apenas no acompanhamento da função auditiva e do desenvolvimento da linguagem oral das crianças de sua região, mas também no reforço da orientação sobre a ocorrência das perdas de audição adquiridas ou de início tardio na população infantil e no resgate das famílias que não retornam para completar o processo de identificação nos Programas de TAN vinculados à maternidade.

\section{AGRADECIMENTOS}

Agradecemos à Fundação de Amparo à Pesquisa do Estado de São Paulo (FAPESP) e ao Conselho Nacional de Desenvolvimento Científico e Tecnológico (CNPq), pelo apoio concedido para realização dessa pesquisa, sob processo número 2003/06415-6 e 403719/2004-6, respectivamente.

\begin{abstract}
Purpose: To evaluate the commitment of parents for carrying out the hearing procedures of a Children's Hearing Health Program at their community. Methods: It was carried out a comparative analysis of the information regarding parents' attendance at two Children's Hearing Health Programs, one developed at a public hospital and the other carried out along with the Family Health Program (FHP). The sample comprised 362 children born between February and March 2007 at the hospital in which the hearing screening was performed, and who were enrolled in the FHP. Results: From the 362 children who had been born at the hospital and referred for hearing evaluation at the basic health unit of the same community, 147 attended the evaluation, representing $40.61 \%$ of commitment to the project developed there. Only $39.80 \%$ of the families whose children's screening suggested no hearing alterations committed the program carried out along with the FHP. Thirty-two families (55.18\%) did not participate in any of the existing hearing health programs, therefore remaining unaware of their child's hearing condition. Conclusion: The attendance of the families at the basic health unit was less than half of the families who had been invited to take the children for hearing evaluation, regardless if the child had been submitted to the process of identification of hearing loss at the public hospital.
\end{abstract}

Keywords: Hearing; Hearing loss; Newborn hearing screening; Family health; Single Health System; Health Program and Plans

\title{
REFERÊNCIAS
}

1. American Academy of Pediatrics, Joint Committee on Infant Hearing. Year 2007 position statement: Principles and guidelines for early hearing detection and intervention programs. Pediatrics. 2007;120(4):898-921.

2. Downs MP, Yoshinaga-Itano C. The efficacy of early identification and intervention for children with hearing impairment. Pediatr Clin North Am. 1999;46(1):79-87. Review.

3. Brasil. Ministério da Saúde. Portaria n 587 de 7 de outubro de 2004. Determina que as Secretárias de Estado da Saúde dos Estados adotem as providências necessárias à organização e implantação das Redes Estaduais de Atenção à Saúde Auditiva. Diário Oficial da União. 11 out 2004; Seção 1: 105.

4. Arnold CL, Davis TC, Humiston SG, Bocchini JA Jr, Bass PF 3rd, Bocchini A, et al. Infant hearing screening: stakeholder recommendations for parentcentered communication. Pediatrics. 2006;117(5 Pt 2):S341-54.

5. Durante AS, Carvallo RMM, Costa MTZ, Cianciarullo MA, Voegels RL, Takahashi GM, et al. A implementação de programa de triagem auditiva neonatal universal em um hospital universitário brasileiro. Pediatria (São Paulo). 2004;26(2):78-84.

6. Hatzopoulos S, Qirjazi B, Martini A. Neonatal hearing screening in Albania: results from an ongoing universal screening program. Int J Audiol. 2007;46(4):176-82.

7. Korres S, Balatsouras DG, Vlachou S, Kastanioudakis IG, Ziavra NV, Ferekidis E. Overcoming difficulties in implementing a universal newborn hearing screening program. Turk J Pediatr. 2005;47(3):203-12.

8. Korres SG, Balatsouras DG, Gkoritsa E, Eliopoulos P, Rallis E, Ferekidis E. Success rate of newborn and follow-up screening of hearing using otoacoustic emissions. Int J Pediatr Otorhinolaryngol. 2006;70(6):1039-43.

9. Low WK, Pang KY, Ho LY, Lim SB, Joseph R. Universal newborn hearing screening in Singapore: the need, implementation and challenges. Ann Acad Med Singapore. 2005;34(4):301-6.

10. Mukari SZ, Tan KY, Abdullah A. A pilot project on hospital-based universal newborn hearing screening: lessons learned. Int J Pediatr Otorhinolaryngol.
2006;70(5):843-51.

11. Barreira-Nielsen C, Futuro Neto HA, Gattaz G. Processo de implantação de Programa de Saúde Auditiva em duas maternidades públicas. Rev Soc Bras Fonoaudiol. 2007;12(2):99-105.

12. Pádua FGM, Marone S, Bento RF, Carvallo RMM, Durante AS, Soares JC, et al. Triagem auditiva neonatal: um desafio para sua implantação. Arq Otorrinolaringol. 2005;9(3):190-4.

13. Abdullah A, Hazim MY, Almyzan A, Jamilah AG, Roslin S, Ann MT, et al. Newborn hearing screening: experience in Malaysian hospital. Singapore Med J. 2006;47(1):60-4.

14. Zhao PJ, Shen XM, Xu ZM, Wu SH, Jin CH, Jiang F. [The parents' opinions on screening program regarding newborn hearing]. Zhonghua Liu Xing Bing Xue Za Zhi. 2003;24(7):608-10. Chinese.

15. Brasil. Ministério da Saúde. Datasus. 2010. Disponível em: http:// w3.datasus.gov.br/datasus/datasus.php.

16. Bevilacqua MC, Alvarenga Kde F, Costa OA, Moret AL. The universal newborn hearing screening in Brazil: from identification to intervention. Int J Pediatr Otorhinolaryngol. 2010;74(5):510-5.

17. Alvarenga KF, Bevilacqua MC, Martinez MANS, Melo TM, Blasca WQ, Taga MFL. Proposta para capacitação de agentes comunitários de saúde em saúde auditiva. Pró-Fono. 2008;20(3):171-6.

18. Rouev P, Mumdzhiev H, Spiridonova J, Dimov P. Universal newborn hearing screening program in Bulgaria. Int J Pediatr Otorhinolaryngol. 2004;68(6):805-10.

19. Brasil. Ministério da Saúde. Secretaria-Executiva. Mais saúde: direito de todos: 2008-2011. Brasília: Ministério da Saúde; 2008. 100 p.

20. Melo TM, Alvarenga KF. Capacitação de profissionais da saúde na área de saúde auditiva: revisão sistemática. Rev Soc Bras Fonoaudiol. 2009;14(2):280-6.

21. Melo TM, Alvarenga KF, Blasca WQ, Taga MLF. Capacitação de agentes comunitários de saúde em saúde auditiva: efetividade da videoconferência. Pró-Fono. 2010; 22(2):139-45. 